\title{
A Literature Review of the Detection and Categorization of various Arecanut Diseases using Image Processing and Machine Learning Approaches
}

\author{
Puneeth B. R. ${ }^{1,2^{*}}$ \& Nethravathi P. S. ${ }^{3}$ \\ ${ }^{1}$ Research Scholar, College of Computer Science and Information Science, Srinivas \\ University, Mangaluru, India \\ ${ }^{2}$ Assistant Professor, Department of M.C.A, NMAMIT, Nitte, Karkala, India \\ Orcid-ID: 0000-0003-1985-0411; E-mail: puneethbr9@gmail.com \\ ${ }^{3}$ Professor, College of Computer Science and Information Science, Srinivas University, \\ Mangaluru, India \\ Orcid-ID: 0000-0001-5447-8673; E-mail: nethrakumar590@gmail.com
}

Subject Area: Computer Science.

Type of the Paper: Review based Research Agenda.

Type of Review: Peer Reviewed as per $|\mathrm{C}| \mathrm{O}|\mathrm{P}| \mathrm{E} \mid$ guidance.

Indexed In: OpenAIRE.

DOI: https://doi.org/10.5281/zenodo. 5773853

Google Scholar Citation: IJAEML

\section{How to Cite this Paper:}

Puneeth, B. R., \& Nethravathi, P. S., (2021). A Literature Review of the Detection and Categorization of various Arecanut Diseases using Image Processing and Machine Learning Approaches. International Journal of Applied Engineering and Management Letters (IJAEML), 5(2), 183-204. DOI: https://doi.org/10.5281/zenodo.5773853

International Journal of Applied Engineering and Management Letters (IJAEML) A Refereed International Journal of Srinivas University, India.

Crossref DOI : https://doi.org/10.47992/IJAEML.2581.7000.0112

(C) With Authors.

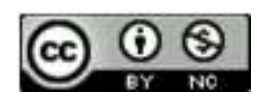

This work is licensed under a Creative Commons Attribution-Non-Commercial 4.0 International License subject to proper citation to the publication source of the work.

Disclaimer: The scholarly papers as reviewed and published by the Srinivas Publications (S.P.), India are the views and opinions of their respective authors and are not the views or opinions of the S.P. The S.P. disclaims of any harm or loss caused due to the published content to any party. 


\title{
A Literature Review of the Detection and Categorization of various Arecanut Diseases using Image Processing and Machine Learning Approaches
}

\author{
Puneeth B. R. ${ }^{1,2^{*}}$ \& Nethravathi P. S. ${ }^{3}$ \\ ${ }^{1}$ Research Scholar, College of Computer Science and Information Science, Srinivas \\ University, Mangaluru, India \\ ${ }^{2}$ Assistant Professor, Department of M.C.A, NMAMIT, Nitte, Karkala, India \\ Orcid-ID: 0000-0003-1985-0411; E-mail: puneethbr9@gmail.com \\ ${ }^{3}$ Professor, College of Computer Science and Information Science, Srinivas University, \\ Mangaluru, India \\ Orcid-ID: 0000-0001-5447-8673; E-mail: nethrakumar590@gmail.com
}

\begin{abstract}
Background/Purpose: Every scholarly research project starts with a survey of the literature, which acts as a springboard for new ideas. The purpose of this literature review is to become familiar with the study domain and to assess the work's credibility. It also improves with the subject's integration and summary. This article briefly discusses the detection of disease and classification to achieve the objectives of the study.

Objective: The main objective of this literature survey is to explore the different techniques applied to identify and classify the various diseases on arecanut. This paper also recommends the methodology and techniques that can be used to achieve the objectives of the study.

Design/Methodology/Approach: Multiple data sources, such as journals, conference proceedings, books, and research papers published in reputable journals, were used to compile the essential literature on the chosen topic and collect information from the arecanuts research centre and many farmers in the south Canara and Udupi districts, before narrowing down the literature that is relevant to the research work. The shortlisted literature was carefully assessed by reading each paper and taking notes as appropriate. The information gathered is then examined to identify the potential gap in the study.
\end{abstract}

Findings/Result: Based on the analysis of the papers reviewed, discussion with farmers and research center officers, it is observed that, not much work is carried out in the field of disease identification and classification on arecanut using machine learning techniques. This survey paper recommends techniques and the methodology that can be applied to identify and classify the diseases in arecanut and to classify them in to healthy and unhealthy.

Research limitations/implications: The literature review mentioned in this paper are detection and classification of different diseases in arecanut.

Originality/Value: This paper focuses on various online research journals, conference papers, technical books, and web articles.

Paper Type: Literature review paper on techniques and methods used to achieve the objectives.

Keywords: Arecanut diseases, Koleroga, Detection of arecanut diseases, Categorization of arecanut diseases, Segmentation of arecanut diseases, Machine learning, Image processing.

\section{INTRODUCTION :}

The seed of the areca palm is known as an arecanut, available in most throughout much of the tropical Pacific-ocean, South-East and South-Asian country, and Eastern Africa place. Betel nut is the common name for it. Areca is derived from the Malayalam word aaykka, it was used by Dutch and Portuguese sailors who imported the nut from India to Europe in the 16th century [1]. It is widely used in India by a vast number of people and is closely associated with religious traditions. In India, the arecanut is a commercial crop. India is the largest producer and eater of arecanut in the world. Karnataka (40 percent), Kerala (25 percent), Assam (20 percent), Tamilnadu, Meghalaya, and West-Bengal are among the states 
that grow this crop [2]. Karnataka is the larger producer of arecanut in India mainly in Dakshina Kannada and Uttara Kannada district and Malnad regions, now days Tumkur, Davangere, Hassan, Mysore districts cultivate arecanut. It thrives in the $14^{\circ} \mathrm{C}$ to $36^{\circ} \mathrm{C}$ temperature range but is harmed by temperatures below $10 \mathrm{oC}$ and above $40 \mathrm{oC}$. Temperature extremes and large diurnal changes are not favorable to healthy palm growth. Arecanuts can be farmed in places with annual rainfall ranging from $750 \mathrm{~mm}$ in Maidan to 4,500 $\mathrm{mm}$ in Malnad in Karnataka. The palms are irrigated in locations where there is a lengthy dry period. A decent harvest of arecanut cannot be obtained at an altitude of more than $1000 \mathrm{~m}$ MSL due to its susceptibility to low temperatures [3]. Gravelly laterite soils of the red clay type have the most area under agriculture. It may also be grown on clay loam soils that are productive. Arecanuts cannot be grown in sticky clay, sandy, alluvial, brackish, or calcareous soils. The arecanut can be used to make diabetes formulations, Ayurveda medication, soaps, areca tea, teeth powder, and wines, in addition to chewing it [4][5]. Koleroga, Bud rot, Inflorescence die back, Yellow leaf disease, Anabe roga/foot rot, etc these all are common diseases on arecanut, these cause significant losses of arecanut tree, bunches, nuts. The Koleroga is the main disease of arecanut [6], Spraying the bunches with a 1 percent Bordeaux mixture before the monsoon, followed by another spray after 40 days, is successful in preventing the disease. Another option is to cover the bunches with plastic bags [7].

\section{RESEARCH OBJECTIVES :}

The objectives play a vital role in providing a detailed view of the goal of any research. The following are the objectives of this literature survey.

- To determine the different types of diseases on arecanut.

- To study the methodology and technology used to identify the diseases of arecanut.

- To know the present status of research on arecanut disease.

- To determine the research gaps and to propose the ideal solution to those research gaps.

- To list out the research agendas and limitations of the research.

- To analyze the proposed agenda using SWOC analysis.

\section{METHODOLOGY :}

Required data and information's are collected from different sources such as books, published research papers, journals, conference papers. The initial information related to arecanut and arecanut diseases was collected from farmers and arecanut research center vittala.

\section{LITERATURE REVIEW :}

Vinod Kanan L. et al., [8] proposed a disease prediction system by using temperature, rainfall, humidity, wind flow soil moisture around the agriculture area and developed a model to predict the diseases. He has summarized the study by concluding the occurrence of disease by analyzing different relationships among environmental factors.

The following papers are related to detecting and classification of arecanut using various machine learning techniques:

Mallikarjuna S. B. et al., [9] proposed a CNN based algorithm to classify the different diseases on arecanut images. He has classified different diseases like rot, split, and rot-split. Results from the fourclass dataset show that the suggested strategy outperforms others in terms of classification rate, recall, precision, and F-measures.

Ajith Danthiz. [10], has developed a novel approach for arecanut classification based on the color of the arecanut, it was divided into two classes. The method was proposed in three different steps: segmentation, masking, and classification, according to the authors. For appropriate segmentation of arecanuts, three sigma control limits are applied to the image. He categorized the arecanut based on two different color like green and red color components of the segmented section. The proposed strategy is more efficient, according to an experimental outcome.

Santhos et al., [11] did the survey paper on different plants disease including arecanut leaf and various advanced techniques uses to detect diseases. In this paper the author has mentioned about different diseases on arecanut plant-like koleroga and yellow leaf. Further he added the cause and effect of the diseases.

Kuo-Yi Huang., [12] grade of arecanuts was discovered and classified. A detection line (DL) method was used to segment the illnesses of arecanuts. In the classification technique, six geometric features, 
three-color features, and the area of the fault were used. A back-propagation neural network classifier was used to sort the quality of arecanuts. This method was provided for classifying arecanuts with a 90.9 percent accuracy.

Rajendra B. et al. [13], has recognized and categorized arecanuts using neural networks and image processing techniques. A back-propagation cellular network classifier was employed to determine the relevance of the arecanut. Various pathogens, such as crops, bacteria, viruses, and insect damage, frequently attack the arecanut. The object color is depicted using the HSI technique, and the contrast curve enhances brightness changes equally over the image's dynamic range. Machine Vision Technology can be used to replace manual sorting in the arecanut advertising industry.

Akshay S. et al. [14], Used husk arecanut photos as input, categorizing healthy and harmful arecanuts. Background subtraction is used in the classification a method for removing shadow effects from pictures. They use the Otsu method to locate the diseased area of the arecanut. To identify the images, the author employs the GLCM texture feature extraction approach and a decision tree. This approach is developed in MATLAB and yields a $90 \%$ accuracy rate.

Anilkumar M. G. et al. [15], detected a system of diseases of arecanut, leaves, and its trunk using Convolutional Neural Networks and suggests remedies for it. A Convolutional Neural Network (CNN) is a Deep Learning algorithm used to detect the diseases of arecanut, that takes input as an image, assigns learnable weights and biases to various objects in the image, and then learns from the results to distinguish one from the other. To train and test the CNN model, created their dataset which has 620 images of arecanut both healthy and diseased. The train and test data are divided into an 80:20 ratio. For a compilation of the model categorical cross-entropy is used as loss function with adam as optimizer function and accuracy as metrics. A total of 50 Epochs are used to train the model to achieve high validation and test accuracy with minimum loss. The proposed approach was found to be effective and has 88.46 percent accuracy for detecting the arecanut disease.

Following papers are related to grading and quality of raw arecanut:

Siddesha S. et al. [16], have applied the KNN algorithm to find diseases using color histograms and color moments as features, and he focused on raw arecanut. An experiment is constructed on a dataset of 800 images of four classes using two-color features and four distance measurements with KNN. After 20 percent training, a classification accuracy of 98 percent is achieved with a $\mathrm{K}$ value of 3 and a Euclidean distance measure for color histogram features.

Suresh M. et al. [17]. proposed textural features of Local Binary Pattern (LBP), Haar Wavelets, GLCM, and Gabor used to identify diseases arecanut and undiseases arecanut. These procedures were carried out in two steps. (1) Each color component of the HSI and YCbCr color models has been treated with LBP. (2) A LBP histogram is created. The rate of success was 92.00 percent. The results were bad in the first stage, thus texture characteristics from Haar wavelets, GLCM, and Gabor were applied in the second stage. The RGB input arecanut image is converted to HSI and $\mathrm{YCbCr}$ color models in this stage, and texture details from each color component are extracted. The applied KNN classifier has a 100\% success rate on a discriminative subset of texture.

Bharadwaj $\mathrm{N} \mathrm{K}$ et al. [18], classified and graded the arecanut by using image processing and computer vision. The author uses color, size, and texture to classify the grading of arecanut. The major goal of this article is to give a comprehensive overview of arecanut, Computer Vision, and the needs and uses of vision-based technology in arecanut categorization and grading.

Dhanuja K. C. et al. [19], proposed a technique for detecting arecanut disease using image processing technology, and the author used texture-based grading of arecanut. To extract numerous textural features from arecanut, Wavelet, Gabor, Local binary (LBP), Gray Level Difference Matrix (GLDM), and Gray Level Co-Occurrence Matrix (GLCM) are employed. A total of 144 arecanut samples were utilized for training and testing the model, with 49 Good, 46 Poor, and 49 Negative samples, and the K N N algorithm was employed to detect illnesses in arecanut. The average accuracy was 90.9 percent.

Siddesh et al. [20], using K-Means and the Otsu approach, a disease detection model was presented to detect and identify afflicted raw arecanut. The arecanut image is segregated from the backdrop using color K-means clustering in preprocessing to remove shadow effects. The RGB image is transformed to monochrome using Otsu thresholding in illness detection. The connected components approach is then used to designate the afflicted arecanut regions. There were 50 disease-affected raw arecanut photos in the dataset. Counting the number of spots in the RGB image and then counting the number of spots spotted in the tagged images are used to evaluate the model's performance. 
Bharadwaj N K [21] proposed to find the grade of the arecanut by using arecanut images. A proposed method for future extraction used the local binary pattern method. Support vector machine-classified used to find the grade of the arecanut. The accuracy, precision, recall, and $\mathrm{F}$-measure generated from the confusion matrix are all used to evaluate the grading system's performance.

Siddesh et al. [22], proposed a texture grading method for arecanut. The textural features of arecanut are extracted using Wavelet, Gabor, Local Binary Pattern (LBP), Gray Level Difference Matrix (GLDM), and Gray Level Co-Occurrence Matrix (GLCM) features. The arecanuts were classified using the Nearest Neighbor (NN) classification technique. The suggested model's performance was demonstrated using 700 arecanut photos of seven distinct classes in an experiment, and the classification rate was 91.43 percent utilizing Gabor wavelet features.

Ajit Danti [23] proposed segmentation techniques and classification methods of raw arecanut. The author has developed a novel approach for categorizing arecanuts into two classes based on color. I Segmentation, (ii) Masking, and (iii) Classification. The classification is based on different two colors like red and green components of the arecanut segmented region. The categorization success rate in the experimental efficiency was around 97 to 98 percent.

Ajith Danti et al. [24], proposed the effective grading of arecanuts. The RGB image of the arecanut is transformed to YC B C R colour space. Three sigma control limits on colour characteristics were identified for successful segmentation of arecanuts. Arecants are graded using the support vector machine (SVM) method and colour features. Boiling and non-boiling nuts are efficiently graded, and experimental findings show that the suggested strategy is effective when using the k-fold crossvalidation method.

Ajit Danti et al. [25], discussed techniques for grading arecanuts based on textural properties. Mean around features, Gray level co-occurrence matrix (GLCM) features, and combination (Mean aroundGLCM) features are used for classification. Arecanuts are classified into six classes using a decision tree classifier. In Decision trees classifier used GLCM features and Mean Around-GLCM features. Testing is conducted by using the cross-validation method. The experimental outcome is a $97.65 \%$ success rate in GLCM features, a 98.28 percent success rate in Mean Around features, and a 99.05 percent success rate in Mean Around-GLCM features.

Sameer Patil et al. [26], proposed a technique for quality classification of arecanut using preprocessing techniques. A Raspberry-Pi board and a 5 Megapixel camera module are used to capture the images. Image filtering, contrast enhancement, and an image segmentation algorithm are all used in the pre-processing of the acquired image. Canny edge detection and the K-means segmentation method were used to detect the nut's boundary while cropping the arecanut photos. In this research, the author categorized eight distinct image pre-processing algorithms to produce the best results for arecanut segregation method.

Ajit Danti et al. [27], proposed a grading system of arecanut. The author combined two algorithms KNN and SUV to classify the nuts for better results.

The following papers are related to robot system arecanut farming:

Devang et al. [28], demonstrated the design and development of an arecanut farming-focused autonomous tree climbing and trimming robot. The robot has a non-linear self-regulatory system based on linear frictional force. The robot's chassis is designed to be both sturdy and light. The power source is an onboard battery. In a Digital Image Processing System, Artificial Intelligence is built on Object Recognition techniques. A guiding camera is installed over the Robotic Arm processor, which incorporates a Wavelet-based JPEG compression and noise reduction module for machine vision. When necessary, a display panel is used to view and control the robot.

S Kushal Gowda et al. [29], designed a smart arecanut plunking robot to climb the arecanut tree. The robot has a camera that gets the video stream through Raspberry pi. Android application is used so the user can watch the video stream. The arecanut bunch was successfully tested by the robot, and the operator was able to successfully lead the robot in cutting the ripened arecanut bunch.

The following paper is related to detecting bunches using various techniques:

Dhanesha R. et al. [30], Used the HSV color model, determine and identify the automated maturity level of arecanut bunches. Experiments were carried out on 200 photographs of arecanut bunches of varying ripeness degrees. The end findings of the investigation reveal that segments arecanut bunches photographs with an accuracy of 85 to $90 \%$. The VOE and DSC segmentation performance matrices are used to assess the precision with which results are obtained. 
Dhanesha R. et al. [31], identified the maturity level of arecanut bunches. The $\mathrm{YCgCr}$ color model automatically segments the arecanut bunch from a given image, using this segment image could determine the arecanut maturity level. The author has created a 1000 images database and captured them by mobile camera. The experimental result shows from this method for associate input image with an accuracy of $80 \%$.

Dhanesha et al. [32], provided dynamic contouring segmentation, in which segments arecanut bunches in pictures. The author gathered 20 photographs of arecanut bunches at various stages of ripeness. VOE and DSC performance measures were used to assess the accuracy of the results. There are no published benchmark results to which the proposed method may be compared for efficiency. For low-quality photos, the proposed approach may not segment well. With the goal of determining the ripeness of arecanut bunches, the author presented a computer vision-based approach for segmentation utilizing active contouring.

Siddesha et al. [33], have segmented of oil palm crop bunch images using supervised and unsupervised approaches. The author used Hill climbing, Growcut, Random Walker, MSRM algorithms for supervised techniques, and K-Means, Fuzzy-C-Means algorithms for unsupervised techniques on a dataset of 100 bunch photos. If apply the same techniques to arecanut bunch would be getting a better result.

Hubert Cecotti et al. [34], have detected grape bunches using a convolution neural network. The different segmentation techniques use to segment the two types of grapes that are white and red grape bunches then apply the CNN algorithm it performed 99\% accuracy.

Scarlett Liu et al., [35] proposed a grape bunch detection system from the image. The image processing and support-vector machine algorithm is used to count the grape bunches from images, and the overall success rate is $88 \%$ for red grape.

André Silva Aguiar et al. [36], proposed detection of the grape bunch in different growing stages using different image-processing and deep-learning models. The dataset has 1929 images with different growth stages of the bunch. The results showed difficulty to detect the bunches in the early and middle growing stage overall $66.96 \%$ detection accuracy.

Lucas Mohimon et al. [37], have been compared deep-learning and machine-learning techniques for grape bunches cluster segmentation. In dataset had 200 images of white color grape in normal light condition and reached $86 \%$ of accuracy. The results showed that deep-learning techniques are more robust to white color grape bunch detection compared with classic segmentation techniques.

Ashfaqur Rahman et al. [38], presented a sequence of image processing and intelligence process used to find the mature grape bunches. The entire process is divided into 2 steps, the grape bunches images are separated from the background image in the first step, wherein the second step the classify the grape bunch based on the mature group, and overall achieved $96 \%$ of accuracy.

Table 1: Related papers on disease detection, quality, and grading on different fruits and vegetables

\begin{tabular}{|c|c|c|c|c|c|c|}
\hline S. & Author & Year & Method & Plant & Finding & Results \\
\hline 1 & $\begin{array}{l}\text { Asmaa Ghazi } \\
\text { Alharbi et al., } \\
\text { [39] }\end{array}$ & 2020 & CNN & Apple & \begin{tabular}{|lcr} 
Detected & and classified \\
different & diseases & on \\
apples & &
\end{tabular} & $\begin{array}{l}\text { Good } \\
\text { classification } \\
\text { accuracy on } \\
90 \% \text { on testing } \\
\text { images }\end{array}$ \\
\hline 2 & $\begin{array}{l}\text { Prasad et al., } \\
\text { [40] }\end{array}$ & 2012 & SVM & crop & $\begin{array}{l}\text { Detected various diseases } \\
\text { on different crop }\end{array}$ & Around $89 \%$ \\
\hline 3 & $\begin{array}{l}\text { Arivazhagan } \\
\text { et al., [41] }\end{array}$ & 2013 & S.V.M & $\begin{array}{l}\text { Different fruits } \\
\text { and vegetables } \\
\text { like Lemon, } \\
\text { Mango } \\
\text { Jackfruits } \\
\text { Banana Beans, } \\
\text { Potato and } \\
\text { Tomato }\end{array}$ & $\begin{array}{ll}\text { - } & \text { Late-Scorch } \\
\text { - } & \text { Bacterial and } \\
& \text { Fungal-Spot } \\
\text { diseases } \\
\text { - } & \text { Sun-Burn } \\
\text { - } & \text { Sooty-Mold } \\
\text { - } & \text { Early and Late- } \\
& \text { Blight } \\
\text { - Scorch Ashen- } \\
\text { Mold }\end{array}$ & $94.74 \%$ \\
\hline
\end{tabular}




\begin{tabular}{|c|c|c|c|c|c|c|}
\hline & & & & & - $\quad$ Leaf-Lesion & \\
\hline 4 & $\begin{array}{l}\text { Kiran Gavhale } \\
\text { et al., [42] }\end{array}$ & 2014 & $\begin{array}{l}\text { S.V.M-RBF } \\
\text { and } \\
\text { S.V.M- } \\
\text { POLY }\end{array}$ & Citrus & $\begin{array}{l}\text { Citrus-Canker, } \\
\text { Anthracnose }\end{array}$ & $96 \%$ and $95 \%$ \\
\hline 5 & $\begin{array}{l}\text { Singh and } \\
\text { Mishra [43] }\end{array}$ & 2016 & $\begin{array}{l}\text { Kmean } \\
\text { and GA, } \\
\text { SVM with } \\
\text { GA }\end{array}$ & $\begin{array}{l}\text { Banana Beans } \\
\text { Lime Rose }\end{array}$ & $\begin{array}{ll}\text { - } & \text { Bacterial and } \\
& \text { Frog-Eye Leaf- } \\
& \text { Spot } \\
\text { - } & \text { Sun-Burn } \\
\text { - } & \text { Early-Scorch }\end{array}$ & $\begin{array}{l}86.54,93.63 \% \\
\text { and } 95.71 \%\end{array}$ \\
\hline 6 & $\begin{array}{l}\text { Jayamoorthy } \\
\text { and Palanivel } \\
\text { [44] }\end{array}$ & 2017 & $\begin{array}{l}\text { Spatial } \\
\text { Fuzzy C } \\
\text { Mean } \\
\text { (SFCM), } \\
\text { SVM } \\
\end{array}$ & Plants & $\begin{array}{l}\text { Bacterial Blight, } \\
\text { Foot Rot }\end{array}$ & Better accuracy \\
\hline 7 & $\begin{array}{l}\text { Mokhtar et al., } \\
\text { [45] }\end{array}$ & 2015 & $\begin{array}{l}\text { S.V.M with } \\
\text { Cauchy } \\
\text { kernel, }\end{array}$ & Tomato & $\begin{array}{l}\text { Powdery-Mildew and } \\
\text { Early-Blight }\end{array}$ & $99.5 \%$. \\
\hline 8 & $\begin{array}{l}\text { Omrani et al., } \\
\text { [46] }\end{array}$ & 2014 & $\begin{array}{l}\text { SVR-RBF, } \\
\text { SVR- } \\
\text { POLY, } \\
\text { ANN }\end{array}$ & Apple & $\begin{array}{l}\text { Alternaria, } \\
\text { Apple Black-Spot, Apple } \\
\text { Leaf-Miner }\end{array}$ & $\begin{array}{l}\text { SVR } \\
\text { outperforms } \\
\text { ANN. }\end{array}$ \\
\hline 9 & $\begin{array}{l}\text { Yadav } \\
\text { and } \\
\text { Verma [47] }\end{array}$ & 2016 & BPNN, GA & Tomato & Black-Mold & $\begin{array}{l}\text { Improved } \\
\text { precision }\end{array}$ \\
\hline 10 & $\begin{array}{l}\text { Bhog and } \\
\text { Pawar [48] }\end{array}$ & 2016 & $\begin{array}{l}\text { Neural- } \\
\text { Network }\end{array}$ & Cotton & $\begin{array}{l}\text { - Red, White and } \\
\text { Yellow-Spot } \\
\text { - Alternaria } \\
\text { - Cercospora }\end{array}$ & $\begin{array}{l}89.55 \% \\
\text { accuracy }\end{array}$ \\
\hline 11 & $\begin{array}{l}\text { Suresha et } \\
\text { al [49] }\end{array}$ & 2017 & K.N.N & Plant & Disease's detection & $76.59 \%$ \\
\hline 12 & $\begin{array}{l}\text { Hossain et } \\
\text { al [50] }\end{array}$ & 2019 & K.N.N & Plant & $\begin{array}{ll}\text { - } & \text { Alternaria- } \\
& \text { Alternate, and } \\
& \text { Anthracnose } \\
\text { - } & \text { Bacterial-Blight } \\
\text { - } & \text { Leaf-Spot } \\
\text { - } & \text { Canker } \\
\end{array}$ & $96.76 \%$ \\
\hline 13 & $\begin{array}{l}\text { Abdhulridha } \\
\text { et al., [51] }\end{array}$ & 2019 & $\begin{array}{l}\text { KNN and } \\
\text { MLP }\end{array}$ & Avacoda & 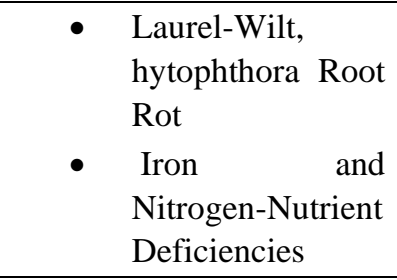 & $\begin{array}{l}\text { Inaccuracy } \\
\text { MLP } \\
\text { outperformed } \\
\text { KNN }\end{array}$ \\
\hline 14 & Ananthi N [52] & 2019 & $\begin{array}{l}\text { DNN } \\
\text { classificatio } \\
\mathrm{n}\end{array}$ & Mango & fungus. & $\begin{array}{l}\text { DNN } \\
\text { classification is } \\
\text { a good way to } \\
\text { find out the } \\
\text { diseases. }\end{array}$ \\
\hline 15 & $\begin{array}{l}\text { Nikhitha } \mathrm{M} \text { et } \\
\text { al., [53] }\end{array}$ & 2019 & $\begin{array}{l}\text { Inception } \\
\text { V3 Model }\end{array}$ & $\begin{array}{l}\text { banana, apple, } \\
\text { and cherry }\end{array}$ & $\begin{array}{l}\text { Level of the disease and } \\
\text { grades }\end{array}$ & $\begin{array}{l}\text { More than } 90 \% \\
\text { accuracy }\end{array}$ \\
\hline 16 & $\begin{array}{l}\text { HongJun Wang } \\
\text { et al., [54] }\end{array}$ & 2020 & R-CNN & $\begin{array}{l}\text { Apple } \\
\text { Peach } \\
\text { Orange, } \\
\text { Pear }\end{array}$ & Detecting disease spots & $\begin{array}{l}\text { Detection } \\
\text { accuracy-95\% } \\
\text { detection speed- } \\
2.6 . \\
\text { FastR-CNN and } \\
\text { SSD algorithms } \\
\text { are not superior }\end{array}$ \\
\hline
\end{tabular}




\begin{tabular}{|c|c|c|c|c|c|c|}
\hline 17 & $\begin{array}{l}\text { Mrunmayee } \\
\text { Dhakate [55] }\end{array}$ & 2015 & ANN & Pomegranate & $\begin{array}{l}\text { Detecting Bacterial-Blight, } \\
\text { Fruit and Leaf spot. }\end{array}$ & $\begin{array}{l}\text { More than } 90 \% \\
\text { accuracy }\end{array}$ \\
\hline 18 & $\begin{array}{l}\text { Dhapitha } \\
\text { Nesarajan et } \\
\text { al., [56] }\end{array}$ & 2020 & $\begin{array}{l}\text { SVM } \\
\text { CNN }\end{array}$ & coconut & $\begin{array}{l}\text { detection of insect } \\
\text { infestation and nutrient } \\
\text { shortage in coconut leaves, } \\
\text { as well as disease analysis }\end{array}$ & $\begin{array}{l}\text { SVM } 93.54 \% \text {, } \\
\text { CNN } 93.72 \% \text { of } \\
\text { accuracy }\end{array}$ \\
\hline 19 & $\begin{array}{l}\text { Abjirami S. et } \\
\text { al., [57] }\end{array}$ & 2019 & $\begin{array}{l}\text { Neural } \\
\text { Network }\end{array}$ & Fruits & $\begin{array}{l}\text { Separate the bacterial and } \\
\text { fungal diseases into } \\
\text { categories. }\end{array}$ & $\begin{array}{l}\text { Bacterial-92\% } \\
\text { Fungal-86\% of } \\
\text { accuracy }\end{array}$ \\
\hline 20 & $\begin{array}{l}\text { Bhavini J et al., } \\
{[58]}\end{array}$ & 2016 & $\begin{array}{l}\text { Random } \\
\text { forest } \\
\text { classifier }\end{array}$ & Apple & Classify the diseases & $\begin{array}{l}\text { The diseases are } \\
\text { recognized } \\
\text { using such a } \\
\text { random forest } \\
\text { classifier and } \\
\text { Feature's level } \\
\text { fusion gives } \\
\text { better accurate }\end{array}$ \\
\hline 21 & $\begin{array}{l}\text { Eduardo } \\
\text { Assunção et al., } \\
\text { [59] }\end{array}$ & 2020 & $\mathrm{CNN}$ & Peach & $\begin{array}{l}\text { Determine the three types } \\
\text { of peach diseases and how } \\
\text { to classify the healthy } \\
\text { peach fruits. }\end{array}$ & $\begin{array}{l}\text { The suggested } \\
\text { model has a } \\
\text { Macro-average } \\
\text { F1-score of } \\
0.96 \text {. }\end{array}$ \\
\hline 22 & $\begin{array}{l}\text { Santi Kumari } \\
\text { Behera et al., } \\
{[60]}\end{array}$ & 2018 & $\begin{array}{l}\text { multi-class } \\
\text { SVM with } \\
\text { K-means } \\
\text { clustering }\end{array}$ & orange & $\begin{array}{l}\text { Classify the different } 4 \\
\text { types of disease }\end{array}$ & $90 \%$ \\
\hline 23 & $\begin{array}{l}\text { M. K. Monir } \\
\text { Rabby et al., } \\
{[61]}\end{array}$ & 2018 & $\begin{array}{l}\text { CED } \\
\text { MCED }\end{array}$ & $\begin{array}{l}\text { Apple } \\
\text { Orange }\end{array}$ & $\begin{array}{l}\text { Color and shape to detect } \\
\text { and classify the fruits }\end{array}$ & $\begin{array}{l}\text { MCED is better } \\
\text { than CED }\end{array}$ \\
\hline 24 & $\begin{array}{l}\text { B. Doh et al., } \\
{[62]}\end{array}$ & 2019 & $\begin{array}{l}\text { K-mean } \\
\text { SVM } \\
\text { CNN }\end{array}$ & citrus & $\begin{array}{l}\text { Detected and classified } \\
\text { diseases using their } \\
\text { physical attributes. }\end{array}$ & $\begin{array}{l}\text { CNN gives } \\
\text { better accuracy }\end{array}$ \\
\hline 25 & $\begin{array}{l}\text { H. Al-Hiary et } \\
\text { al., [63] }\end{array}$ & 2012 & $\begin{array}{l}\text { K-means } \\
\text { clustering } \\
\text { Multi-class } \\
\text { SVM } \\
\end{array}$ & Apple & $\begin{array}{l}\text { detect and classify the } \\
\text { examined diseases }\end{array}$ & Accuracy 93\% \\
\hline 26 & $\begin{array}{l}\text { Bargoti et al., } \\
{[64]}\end{array}$ & 2017 & $\begin{array}{l}\text { Faster R- } \\
\text { CNN VGG } \\
\text { and R-CNN } \\
\text { ZF } \\
\end{array}$ & $\begin{array}{l}\text { mangoes, } \\
\text { almonds, } \\
\text { apples }\end{array}$ & Fruit detection & $\begin{array}{l}\text { R-CNN VGG } \\
\text { has given a best } \\
\text { performance } \\
\text { than R-CNN ZF }\end{array}$ \\
\hline 27 & $\begin{array}{l}\text { Ramesh Kestur } \\
\text { et al., [65] }\end{array}$ & 2019 & $\begin{array}{l}\text { Fully } \\
\text { convolution } \\
\text { al networks }\end{array}$ & Mango & $\begin{array}{l}\text { In RGB photos, mangoes } \\
\text { are detected and counted. }\end{array}$ & $\begin{array}{l}\text { The better result } \\
\text { with good } \\
\text { accuracy }\end{array}$ \\
\hline 28 & $\begin{array}{l}\text { Ashwini Awate } \\
\text { et al., [66] }\end{array}$ & 2015 & ANN & $\begin{array}{l}\text { grape, apple, } \\
\text { pomegranate }\end{array}$ & $\begin{array}{l}\text { Classification of disease by } \\
\text { using colour }\end{array}$ & $\begin{array}{l}\text { Reduces } \\
\text { Human efforts } \\
\text { and } \quad 90 \% \\
\text { accurate } \\
\text { classification. }\end{array}$ \\
\hline 29 & $\begin{array}{l}\text { P. Prathusha et } \\
\text { al., [67] }\end{array}$ & 2020 & $\begin{array}{l}\text { SVM, } \\
\text { Naive } \\
\text { Bayes, } \\
\text { KNN }\end{array}$ & Tomato & $\begin{array}{lll}\begin{array}{l}\text { Detected } \\
\text { diseases }\end{array} & 15 & \text { various } \\
\end{array}$ & $\begin{array}{l}\text { KNN is a best } \\
\text { Machine } \\
\text { learning } \\
\text { algorithm }\end{array}$ \\
\hline 30 & $\begin{array}{l}\text { Harshal } \\
\text { Waghmare et } \\
\text { al., [68] }\end{array}$ & 2016 & $\begin{array}{l}\text { multiclass } \\
\text { SVM }\end{array}$ & grape & $\begin{array}{l}\text { Major diseases of grapes } \\
\text { downy mildew \& black rot } \\
\text { classified }\end{array}$ & $\begin{array}{l}96.6 \% \\
\text { accuracy }\end{array}$ \\
\hline 31 & $\begin{array}{l}\text { Liton Jude } \\
\text { Rozario et al., } \\
\text { [69] }\end{array}$ & 2016 & $\begin{array}{l}\text { Segmentati } \\
\text { on using K- } \\
\text { mean and } \\
\text { modified K- } \\
\text { mean }\end{array}$ & $\begin{array}{l}\text { Apple, banana, } \\
\text { potato, tomato }\end{array}$ & $\begin{array}{l}\text { Identify faulty areas in a } \\
\text { variety of fruits and } \\
\text { vegetables. }\end{array}$ & $\begin{array}{l}\text { The Otsu } \\
\text { technique } \\
\text { performs better } \\
\text { than K-means } \\
\text { clustering. }\end{array}$ \\
\hline
\end{tabular}

Puneeth B. R., et al, (2021); www.srinivaspublication.com 


\begin{tabular}{|c|c|c|c|c|c|c|}
\hline & & & $\begin{array}{l}\text { clustering, } \\
\text { Otsu } \\
\text { method }\end{array}$ & & $\begin{array}{l}\text { Examine various } \\
\text { segmentation approaches } \\
\text { to find the best one. }\end{array}$ & \\
\hline 32 & $\begin{array}{l}\text { Santi Kumari } \\
\text { Behera et al., } \\
{[70]}\end{array}$ & 2021 & $\begin{array}{l}\text { KNN, } \\
\text { SVM, } \\
\text { Naïve } \\
\text { Bayes }\end{array}$ & papaya & $\begin{array}{l}\text { The maturity status using } \\
\text { the classification of the } \\
\text { machine (LBP, HOG, and } \\
\text { GLCM features) and } \\
\text { transfer learning approach. }\end{array}$ & $\begin{array}{lr}\text { KNN } & \text { with } \\
\text { HOG } & \text { better } \\
\text { perform } & \text { with } \\
\text { accuracy } & 100 \%\end{array}$ \\
\hline 33 & $\begin{array}{l}\text { Dor } \\
\text { Oppenheim et } \\
\text { al., [71] }\end{array}$ & 2019 & $\begin{array}{l}\text { Deep } \\
\text { learning }\end{array}$ & Potato & $\begin{array}{l}\text { Identify and classify the } \\
\text { diseases }\end{array}$ & $\begin{array}{l}\text { classification } \\
\text { rates more than } \\
90 \% \text {. }\end{array}$ \\
\hline 34 & 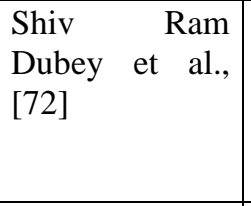 & 2016 & $\begin{array}{l}\text { K-mean, } \\
\text { SUV }\end{array}$ & Apple & $\begin{array}{l}-\quad \text { Apple blotch } \\
\text { - rot and scab } \\
\text { these diseases to detect } \\
\text { and classified. }\end{array}$ & $\begin{array}{l}\text { A combination } \\
\text { of features CCV } \\
+ \text { CLBP }+ \text { ZM } \\
\text { got better } \\
\text { accuracy }\end{array}$ \\
\hline 35 & s. Usha [73] & 2017 & $\begin{array}{l}\text { k-means } \\
\text { clustering } \\
\text { segmentatio } \\
\mathrm{n}\end{array}$ & $\begin{array}{l}\text { Strawberry, } \\
\text { brinjal }\end{array}$ & Present the maturity level & $\begin{array}{l}\text { the threshold } \\
\text { value for } \\
\text { strawberry is } 98 \\
\text { brinjal is } 70\end{array}$ \\
\hline 36 & $\begin{array}{l}\text { Guoxiang Zeng } \\
{[74]}\end{array}$ & 2017 & CNN & $\begin{array}{l}\text { All fruits and } \\
\text { vegetables }\end{array}$ & $\begin{array}{l}\text { Create a test database for a } \\
\text { fruit and vegetable } \\
\text { classification system based } \\
\text { on visual saliency and the } \\
\text { VGG model. }\end{array}$ & $\begin{array}{l}95.6 \% \text { accuracy } \\
\text { in classification }\end{array}$ \\
\hline 37 & $\begin{array}{ll}\text { Gouri } & \text { C. } \\
\text { Khadabadi } & \text { et } \\
\text { al., [75] } & \end{array}$ & 2015 & $\begin{array}{l}\text { Probabilisti } \\
\mathrm{c} \quad \text { Neural } \\
\text { Network }\end{array}$ & Carrot & $\begin{array}{l}\text { Using various image } \\
\text { processing techniques, } \\
\text { identify and classify } \\
\text { illnesses on carrots. }\end{array}$ & $\begin{array}{l}\text { PNN had good } \\
\text { results, with an } \\
80.0 \quad \% \\
\text { recognition } \\
\text { rate. }\end{array}$ \\
\hline 38 & $\begin{array}{l}\text { Santanu } \\
\text { Phadikar et al., } \\
\text { [76] }\end{array}$ & 2013 & $\begin{array}{l}\text { extracted by } \\
\text { developing } \\
\text { novel } \\
\text { algorithms }\end{array}$ & Rice & $\begin{array}{l}\text { Classification diseases in } \\
\text { rice }\end{array}$ & $\begin{array}{l}\text { provides the } \\
\text { best result } \\
\text { compared to } \\
\text { traditional } \\
\text { classifiers. }\end{array}$ \\
\hline 39 & $\begin{array}{lll}\text { Shiv } & \text { Ram } \\
\text { Dubey } & \text { et } & \text { al., } \\
\text { [77] } & & \end{array}$ & 2012 & $\begin{array}{l}\text { Multi-class } \\
\text { Support } \\
\text { Vector } \\
\text { Machine. } \\
\end{array}$ & Apple & $\begin{array}{l}\text { Find and categorized of } \\
\text { different diseases }\end{array}$ & $\begin{array}{l}93 \% \\
\text { classification } \\
\text { accuracy }\end{array}$ \\
\hline 40 & $\begin{array}{l}\text { C. Santhosh } \\
\text { Kumar et al., } \\
{[78]}\end{array}$ & 2021 & $\begin{array}{l}\text { K Means } \\
\text { Clustering } \\
\text { and SVM. }\end{array}$ & Tomato & $\begin{array}{l}\text { A technique for detecting } \\
\text { diseases in vegetables has } \\
\text { been developed. }\end{array}$ & $\begin{array}{l}\text { It has a high } \\
\text { level of } \\
\text { accuracy and } \\
\text { takes less time } \\
\text { to complete the } \\
\text { entire } \\
\text { operation. }\end{array}$ \\
\hline 41 & $\begin{array}{l}\text { Meenakshi } \\
\text { Pawar et al., } \\
\text { [79] }\end{array}$ & 2012 & SVM & Pomegranate & $\begin{array}{l}\text { Pomegranate Fruit Sorting } \\
\text { and Grading }\end{array}$ & Better accuracy \\
\hline 42 & $\begin{array}{l}\text { Ahmad } \quad \text { Loti } \\
{[80]}\end{array}$ & 2021 & $\begin{array}{l}\text { SVM, } \\
\text { RF, } \\
\text { ANN }\end{array}$ & Chili & $\begin{array}{l}\text { quick identification of chili } \\
\text { disease }\end{array}$ & $\begin{array}{l}\text { Best accuracy } \\
92.10 \% \text { with } \\
\text { SVM classifier }\end{array}$ \\
\hline 43 & $\begin{array}{lr}\text { Hafiz } & \text { Tayyab } \\
\text { Rauf et al., [81] }\end{array}$ & 2019 & $\begin{array}{l}\text { Machine } \\
\text { learning } \\
\text { algorithms }\end{array}$ & Citrus & $\begin{array}{l}\text { Detection } \\
\text { classification of different } \\
\text { diseases }\end{array}$ & $\begin{array}{lr}\text { Maintain the } \\
\text { database and } \\
\text { classify } \\
\text { diseases }\end{array}$ \\
\hline 44 & Yao et al., [82] & 2009 & S.V.M & Rice & $\begin{array}{l}\text { Three different diseases } \\
\text { detected on rice leaf. }\end{array}$ & $\begin{array}{l}97.2 \% \\
\text { accuracy }\end{array}$ \\
\hline
\end{tabular}




\begin{tabular}{|c|c|c|c|c|c|c|}
\hline 45 & $\begin{array}{l}\text { Benjamin Doh } \\
\text { et al., [83] }\end{array}$ & 2019 & $\begin{array}{l}\text { SVM } \\
\text { ANN }\end{array}$ & Citrus & $\begin{array}{|lcc|}\text { Fruit } & \text { disease } & \text { detection } \\
\text { based } & \text { on } & \text { physical } \\
\text { characteristics } & \\
\end{array}$ & $\begin{array}{l}\text { SVM achieves a } \\
\text { better } \\
\text { classification } \\
\text { accuracy over } \\
\text { ANN }\end{array}$ \\
\hline 46 & $\begin{array}{l}\text { Pallavi U. Patil } \\
\text { et al., [84] }\end{array}$ & 2020 & $\begin{array}{l}\text { SVM } \\
\text { ANN } \\
\text { CNN }\end{array}$ & Dragon & $\begin{array}{l}\text { To determine the grade, } \\
\text { consider the shape, size, } \\
\text { weight, colour, and } \\
\text { diseases. }\end{array}$ & $\begin{array}{l}\text { Both algorithms } \\
\text { give a better } \\
\text { result. }\end{array}$ \\
\hline 47 & $\begin{array}{l}\text { Fouzia Risdin } \\
\text { et al., [85] }\end{array}$ & 2020 & $\begin{array}{l}\text { Deep } \\
\text { Convolutio } \\
\text { nal Neural } \\
\text { Network }\end{array}$ & $\begin{array}{l}\text { Grape } \\
\text { Green apple } \\
\text { Lemon } \\
\text { Lychee }\end{array}$ & $\begin{array}{l}\text { CNN is utilized as a } \\
\text { framework feature. Fruits } \\
\text { are divided into categories } \\
\text { based on their types, such } \\
\text { as grapes, apples, lemons, } \\
\text { and so on. }\end{array}$ & $\begin{array}{l}\text { CNN } 99.89 \% \\
\text { accuracy }\end{array}$ \\
\hline 48 & $\begin{array}{l}\text { 1Sheril Angel. } \\
\text { J et al., [86] }\end{array}$ & 2021 & CNN & Tomato & $\begin{array}{l}\text { To detect and classify } \\
\text { diseases on tomatoes, a } \\
\text { deep learning algorithm is } \\
\text { used. }\end{array}$ & $\begin{array}{l}\text { achieved the } \\
\text { accuracy of } \\
97.12 \%\end{array}$ \\
\hline 49 & $\begin{array}{l}\text { Mohanapriya S } \\
\text { et al., [87] }\end{array}$ & 2021 & $\begin{array}{l}\text { RNN } \\
\text { CNN }\end{array}$ & Apple & $\begin{array}{l}\text { RNN is used for the } \\
\text { detection } \\
\text { segmentation of diseases. } \\
\text { CNN used to identify the } \\
\text { diseases }\end{array}$ & $\begin{array}{l}\text { CNN algorithm } \\
\text { shows better } \\
\text { accuracy }\end{array}$ \\
\hline 50 & $\begin{array}{l}\text { Malathy } \\
\text { al., [88] }\end{array}$ & 2021 & CNN & Apple & $\begin{array}{l}\text { Deep learning is used to } \\
\text { identify and detect the } \\
\text { diseased part in apple }\end{array}$ & $\begin{array}{ll}97 \% & \text { overall } \\
\text { result } & \end{array}$ \\
\hline 51 & $\begin{array}{l}\text { Marani et al., } \\
\text { [89] }\end{array}$ & 2021 & $\begin{array}{l}\text { Deep } \\
\text { Neural } \\
\text { Network }\end{array}$ & Grape bunch & $\begin{array}{l}\text { Segmentation accuracy and } \\
\text { IoU method are used to } \\
\text { segment images over four } \\
\text { different deep Neural- } \\
\text { Networks to find the } \\
\text { bunches. }\end{array}$ & $\begin{array}{l}\text { VGG19 } \\
\text { accuracy } 80 \% \\
\text { IoU } 45 \% \text { of } \\
\text { accuracy }\end{array}$ \\
\hline
\end{tabular}

The following are the few major image processing and machine learning algorithms used by the authors in recent years.

Table 2: Image Processing and ML algorithms used in different task

\begin{tabular}{|c|c|}
\hline Task & Techniques \\
\hline Image Pre-processing & $\begin{array}{ll}\text { - } & \text { Brightness correction } \\
\text { - } & \text { Gray scale transformation } \\
\end{array}$ \\
\hline Image Segmentation & $\begin{array}{l}\text { - } \quad \text { Thresholding methods } \\
\text { - } \quad \text { K-Means clustering }\end{array}$ \\
\hline Feature Extraction & $\begin{array}{ll}\text { - } & \text { Histogram thresholding } \\
\text { - } & \text { Edge detection approach } \\
\text { - } & \text { Fuzzy approach } \\
\text { - } & \text { Neural network approach }\end{array}$ \\
\hline Disease Detection & $\begin{array}{ll}\text { - } & \text { K-Means } \\
\text { - } & \text { Otsu method } \\
\end{array}$ \\
\hline Disease Classification & $\begin{array}{ll}\text { - } & \text { CNN } \\
\text { - } & \text { Random Forest } \\
\text { - } & \text { Deep Neural Network } \\
\text { - } & \text { KNN } \\
\text { - } & \text { Decision Tree } \\
\text { - } & \text { SVM }\end{array}$ \\
\hline
\end{tabular}




\section{PRESENT STATUS :}

At present, there are not many research activities done on arecanut disease detection, disease classification, and early detection. The existing algorithms are used to identify the arecanut diseases but with less accuracy and much research has given the focus on checking the quality of the raw arecanut and classifying the arecanut based on the quality, size, color, etc.

If identified in the early stage, farmers can take preventive measures. Till date, no algorithms developed and no existing algorithms on the early detection of arecanut diseases. It's very useful for farmers to stop the spreading of other bunches and other arecanut trees and take precautionary measures. There are several illnesses that affect arecanuts, but the most common one appears during the rainy season and spreads rapidly across the crop. The following table gives the descriptions of different types of diseases in arecanut [90][91].

Table 3: Different types of diseases in arecanut [92].

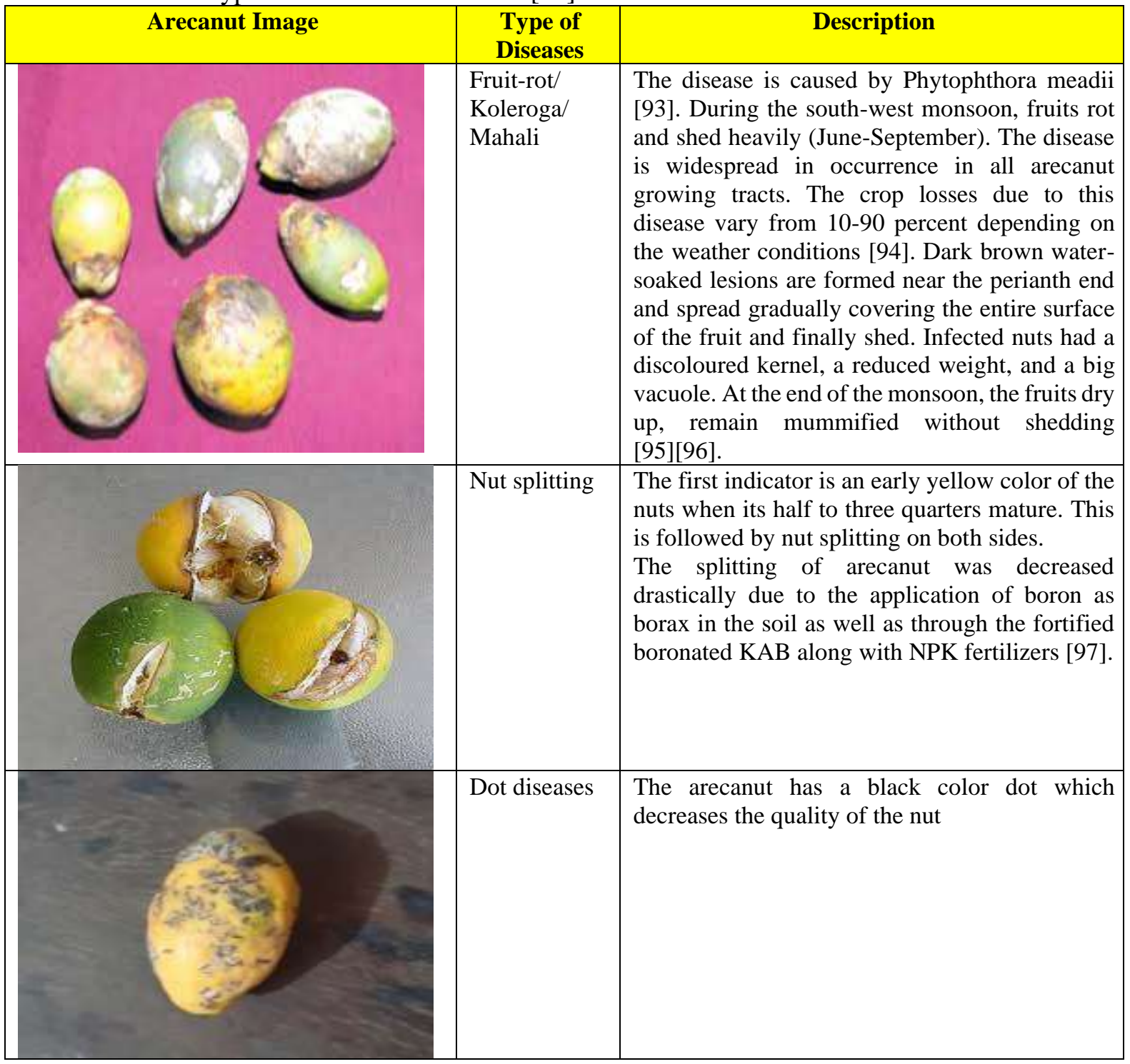




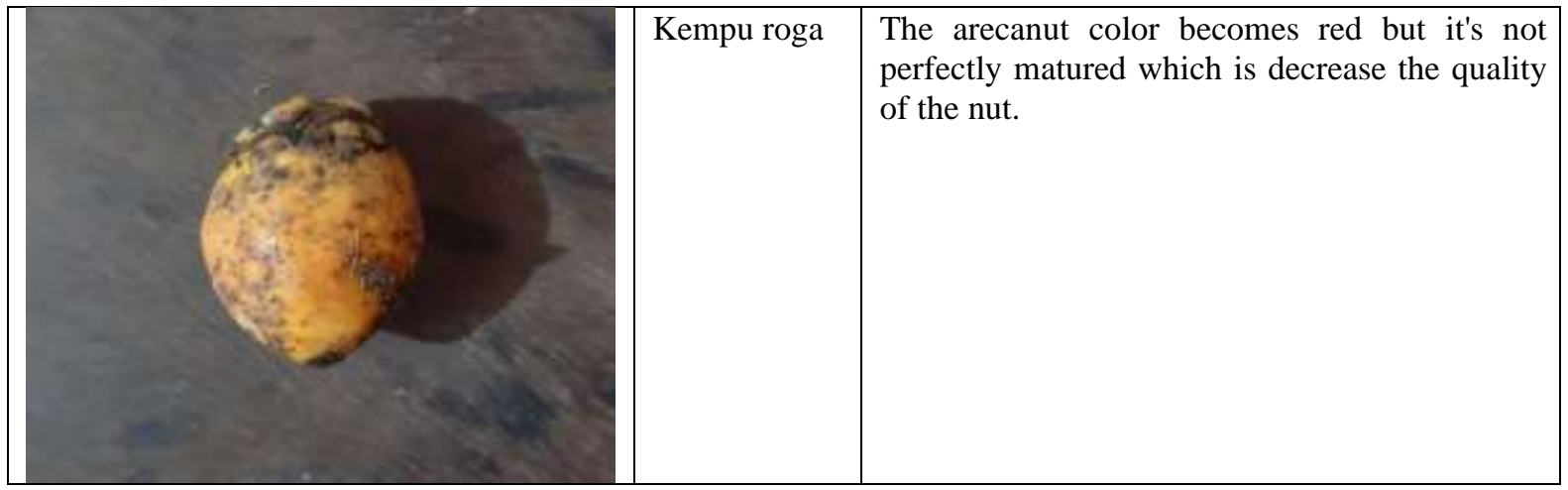

Table 4: Healthy and unhealthy arecanut.

\begin{tabular}{|l|l|l|}
\hline Image & Healthy/Unhealthy & \multicolumn{1}{|c|}{ Description } \\
\hline Healthy & $\begin{array}{l}\text { This is a healthy arecanut } \\
\text { because nut becomes dark } \\
\text { green color. There is no yellow } \\
\text { color surface and black and } \\
\text { white dots }\end{array}$ \\
\hline Unhealthy & $\begin{array}{l}\text { It is an unhealthy arecanut } \\
\text { because initial stage manifest } \\
\text { as dark green or yellowish } \\
\text { water-soaked lesions near the } \\
\text { perianth on the nut surface. } \\
\text { The nut turns a yellowish } \\
\text { colour and has a black surface } \\
\text { [98]. }\end{array}$ \\
\hline & Unhealthy & $\begin{array}{l}\text { It is an unhealthy arecanut, on } \\
\text { the whole surface of the nuts, a } \\
\text { white, black, and brown } \\
\text { mycelial mass envelope them. }\end{array}$ \\
\hline
\end{tabular}

Table 5: Healthy and unhealthy arecanut bunches

\begin{tabular}{|c|c|c|}
\hline \multicolumn{1}{|c|}{ Image } & Healthy/Unhealthy & \multicolumn{1}{c|}{ Description } \\
\hline & Healthy bunch & $\begin{array}{l}\text { This is a healthy arecanut } \\
\text { bunch. There is no yellow, } \\
\text { black, brown color dot or } \\
\text { surfaces. }\end{array}$ \\
\hline
\end{tabular}




\begin{tabular}{|l|l|l|}
\hline Unealthy Bunch & $\begin{array}{l}\text { In the discoloured patches, } \\
\text { the fungus' fruiting bodies } \\
\text { (conidia) appear as } \\
\text { concentric rings. } \\
\text { The disease is more severe } \\
\text { when the weather is dry. }\end{array}$ \\
\hline
\end{tabular}

\section{RESEARCH GAPS AND PROPOSAL :}

After the literature survey, it is observed that the current methodology, models, and publications appear to be lagging in addressing the benefits and challenges of the agriculture area. There is a very insignificant amount of study on the application of technology in the arecanut field. This survey identifies the following research gaps and suggests the solution.

- Research Gap 1: To detect the arecanuts from the arecanut bunch.

- Research Gap 2: To detect and identify the diseases in arecanut.

- Research Gap 3: classify the different types of diseases on arecanut.

- Research Gap 4: Checking the quality of arecanut

- The proposed method can be implemented with SVM, random forests, boosting, fuzzy logic and results can be compared.

- The method has to be enhanced so that it can efficiently classify plants with different types of diseases.

- The percentage of destruction (diseases portion in the plants) can be displayed

\section{RESEARCH AGENDAS :}

1. Which image processing technology is used to pre-process image?

2. How do the segmentation methods to given images using image processing techniques?

3. How to use the feature extraction method to image set?

4. What object detection algorithms can be proposed to find the arecanut from the arecanut bunch?

5. Which machine learning technology platform or algorithms can be used to identify or detect the diseases on arecanut?

6. To find the best machine learning algorithm to detect or identify the diseases in arecanut with good accuracy.

7. What machine learning classification algorithms can be proposed to classify the different types of diseases in arecanut?

\section{LIMITATIONS OF THE PROPOSAL :}

This proposal is only limited to arecanut crop diseases. A similar kind of proposal can be developed for any other crop like coconut or any vegetables or fruits. This proposal only concentrates on arecanut diseases like Kole roga, kempu roga, dot diseases, nut split, etc. A similar kind of disease spreads in arecanut leaf, root, stem, etc. This proposal provides information like whether arecanuts are healthy or unhealthy but never give any solution for diseases and also never predict the diseases.

\section{SWOC ANALYSIS :}


SWOC analysis is primarily concerned with exploring strengths, limitations, opportunities, and challenges in a systematic manner. The SWOC analysis conducted on the research proposal yielded the following results [100][101][102][103].

Table 6: SWOC Analysis

\begin{tabular}{|c|c|}
\hline Strengths & Weaknesses \\
\hline $\begin{array}{ll}\text { - } & \text { Quality product } \\
\text { - } & \text { Use of indigenous technology } \\
\text { - } & \text { Low cost } \\
\text { - } & \text { Controlled diseases spread } \\
\text { - } & \text { Data availability } \\
\end{array}$ & $\begin{array}{ll}\text { - } & \text { Low yield } \\
\text { - } & \text { Prediction of diseases. } \\
\text { - } & \text { Difficult to analyze unstructured data } \\
\text { - } & \text { Lack of quality contents }\end{array}$ \\
\hline Opportunities & Challenges \\
\hline $\begin{array}{ll}\text { - } & \text { Technical Advancements } \\
\text { - } & \text { Increasing market demand } \\
\text { - } & \text { Growing purchase power of consumers } \\
\text { - } & \text { Growing environmental concern }\end{array}$ & $\begin{array}{ll}\text { - } & \text { Control of pest and disease incidence } \\
\text { - } & \text { Climate change } \\
\text { - } & \text { Risk \& uncertainty of marketing } \\
\text { - } & \text { Yield reduction }\end{array}$ \\
\hline
\end{tabular}

\section{CONCLUSION :}

In India, the arecanut is a commercial crop. Many of the farmers of the coastal district Dakshina Kannada, Udupi, Uttara Kannada, malenadu region Shivamoga, Chikmagalur, Davanagere cultivates arecanut, especially for the commercial purpose. Nowadays one of the highest demands and the highest rate of the crop is arecanut. Because of the high rate, almost all Uttara Karnataka and Mysore region farmers also showed interest in arecanut. The arecanut has many diseases because of chemical fertilizer, spraying chemical liquid, climate changes like heavy rain, variation of temperature. The farmers lose the product because of unpredictable diseases. The farmers Spray the arecanut bunches with Bordeaux mixture every year before the monsoon followed by every 40 days, this method follows all farmers for many years. If the farmers know which kind of the diseases and whether the bunch is infected or not before monsoon followed by another spray will be effective in preventing the disease, Covering the bunches with polythene bags is one more method of control. Our goal is to educate the farmers by giving technology touch to find and classify the diseases in arecanut. Our research work concentrates on implementing a system using machine learning and image processing to educate the farmers on different kinds of diseases on arecanut and detecting the diseases inorder to reduce the farmer's work and increase the revenue of the farmer. The proposed model also concentrates on early detection of diseases on arecanut so farmers could be avoided spray in monsoon time. There is a lot of scope for research to understand and find out an effective and reliable subset of technologies contributing to agriculture.

\section{REFERENCES :}

[1] Nair, K. P. (2021). Arecanut (Areca catechu L.). In Tree Crops, 1(1), 1-25. Springer, Cham. Google Scholar X

[2] Mitra, S. K., \& Devi, H. (2016). Arecanut in India-present situation and prospects. In International Symposia on Tropical and Temperate Horticulture-ISTTH2016 1205, 1(1), 789-794.

Google Scholar ${ }^{7}$

[3] Ramappa, B. T., \& Manjunatha, M. S. (2013). Cost cultivation of areca nut non-traditional region of Karnataka-An analysis. International Journal of Pharmaceutical Science Invention, 2(3), 25-31. Google Scholar $x^{7}$

[4] Musdja, M. Y., Nurdin, A., \& Musir, A. (2020). Antidiabetic effect and glucose tolerance of arecanut (Areca catechu) seed ethanol extract on alloxan-induced diabetic male rats. In IOP Conference Series: Earth and Environmental Science, 462(1), 1-9.

Google Scholar ${ }^{\top}$

[5] Arjungi, K. N. (1976). Areca nut: a review. Arzneimittel-forschung, 26(5), 951-956. Google Scholar ${ }^{7}$

[6] Lokesh, M. S., Patil, S. V., Palakshappa, M. G., \& Gurumurthy, S. B. (2014). Role of systemic fungicide metalaxyl mancozeb in management of Koleroga (Phytophthora meadii Mc Rae) of 
arecanut (Areca catechu L.) in Central Western ghats of Karnataka. Asian Journal of Bio Science, 9(1), 131-133.

Google Scholar $\chi^{\nearrow}$

[7] Balanagouda, P., Vinayaka, H., Maheswarappa, H. P., \& Narayanaswamy, H. (2021). Phytophthora diseases of arecanut in India: prior findings, present status, and future prospects. Indian Phytopathology, 1(1), 1-12.

Google Scholar $\chi^{\top} \quad \underline{\text { CrossRefDOI } \chi^{\nearrow}}$

[8] Kanan, L. V et al., (2021). Arecanut Yield Disease Forecast using IoT and Machine Learning. International Journal of Scientific Research in Engineering \& Technology, 2(2), 11-15. Google Scholar ${ }^{\top}$

[9] Mallikarjuna, S. B., Shivakumara, P., Khare, V., Kumar, V., Basavanna, M., Pal, U., \& Poornima, B. (2021). CNN based method for multi-type diseased arecanut image classification. Malaysian Journal of Computer Science, 34(3), 255-265.

Google Scholar ${ }^{\top}$

[10] Ajit Danti., Suresha, (2012). Segmentation and Classification of Raw Arecanuts Based on Three Sigma Control Limits, Procedia Technology. Elsevier Ltd, 4(1),215-219.

Google Scholar $\chi^{\top}$

[11] Kumar, S. S., \& Raghavendra, B. K. (2019). Disease's detection of various plant leaf using image processing techniques: A review. In 2019 5th International Conference on Advanced Computing \& Communication Systems (ICACCS), 1(1), 313-316

Google Scholar $X^{\top}$

[12] Huang, K. Y. (2012). Detection and classification of areca nuts with machine vision. Computers \& Mathematics with Applications, 64(5), 739-746.

Google Scholar ${ }^{\top}$

[13] Rajendra, A. B., Rajkumar, N., \& Shetty, P. D. (2020). Areca Nut Disease Detection Using Image Processing. In Soft Computing: Theories and Applications, 1(1), 925-931.

Google Scholar $x^{7}$

[14] Akshay, S., \& Hegde, A. (2021). Detection and classification of areca nut diseases. In 2021 Second International Conference on Electronics and Sustainable Communication Systems (ICESC), 1(1), 1092-1097.

Google Scholar ${ }^{7}$

[15] Anilkumar M G., Karibasaveshwara TG., Pavan HK., Sainath Urankar., Dr. Abhay Deshpande. (2021). Detection of Diseases in Arecanut Using Convolutional Neural Networks. International Research Journal of Engineering and Technology (IRJET), 8(5), 4282-4286. Google Scholar $\chi^{\top}$

[16] Siddesha, S., Niranjan, S. K., \& Aradhya, V. M. (2018). Color Features and KNN in Classification of Raw Arecanut images. In 2018 Second International Conference on Green Computing and Internet of Things (ICGCIoT), 1(1), 504-509.

Google Scholar $X^{\top}$

[17] Suresha, M., Danti, A., \& Narasimhamurthy, S. K. (2014). Classification of Diseased Arecanut based on Texture Features. International Journal of Computer Applications, 1(1), 1-9.

Google Scholar $\chi^{7}$

[18] Dinesh, R., \& Bharadwaj, N. K. (2017). Possible approaches to arecanut sorting/grading using computer vision: A brief review. In 2017 International Conference on Computing, Communication and Automation (ICCCA), 1(1), 1007-1014.

Google Scholar ${ }^{7}$

[19] Dhanuja, K. C., \& Mohan Kumar, H. P. (2020). Areca Nut Disease Detection using Image Processing Technology. International journal of engineering research \& technology (IJERT), 9(8), 223-226.

Google Scholar $\chi^{7}$

Puneeth B. R., et al, (2021); www.srinivaspublication.com 
[20] Siddesha, S., \& Niranjan, S. K. (2020). Detection of affected regions of disease arecanut using KMeans and Otsu Method. International journal of scientific \& technology research, 9(2), 34043408.

Google ScholarX

[21] Bharadwaj, N. K. (2021). Classification and Grading of Arecanut Using Texture Based Block-Wise Local Binary Patterns. Turkish Journal of Computer and Mathematics Education (TURCOMAT), 12(11), 575-586.

Google Scholar $\chi^{\top}$

[22] Siddesha, S., Niranjan, S. K., \& Aradhya, V. M. (2015). Texture based classification of arecanut. In 2015 International Conference on Applied and Theoretical Computing and Communication Technology (iCATccT), 1(1), 688-692).

Google Scholar $\nearrow^{T}$

[23] Danti, A. (2012). Segmentation and classification of raw arecanuts based on three sigma control limits. Procedia Technology, 4(1), 215-219.

Google Scholar $X^{\top}$

[24] Danti, A., \& Suresha, M. (2012). Arecanut grading based on three sigma controls and SVM. In IEEE-International Conference on Advances in Engineering, Science and ManagementICAESM-2012), 1(1), 372-376.

Google Scholar $X^{\top}$

[25] Danti, A., \& Suresha, M. (2012). Texture based decision tree classification for arecanut. In Proceedings of the CUBE International Information Technology Conference, 1(1), 113-117.

Google Scholar X

[26] Patil, S., Naik, A., Sequeira, M., Naik, G., \& Parab, J. (2021). An Algorithm for Pre-processing of Areca Nut for Quality Classification. In International Conference on Image Processing and Capsule Networks, 1(1), 79-93.

Google Scholar X

[27] Danti, A., \& Suresha, M. (2012). Effective Multiclassifier for Arecanut Grading. In International Conference on Information Processing, 292(1), 350-359. Springer, Berlin, Heidelberg. Google Scholar $x^{\top}$

[28] Devang, P. S., Gokul, N. A., Ranjana, M., Swaminathan, S., \& Binoy, B. N. (2010). Autonomous arecanut tree climbing and pruning robot. In INTERACT-2010, 1(1),278-282.

Google Scholar X

[29] Gowda, S. K., Prathima, K. R., Yashaswini, L., Rakesh, M. D., \& Kumar, T. S. (2019). Pneumatic Controlled Smart Arecanut Plucking Robot with Raspberry Pi. In 2019 International Conference on Communication and Electronics Systems (ICCES), 1(1), 527-532.

Google Scholar $\not$ '

[30] Dhanesha, R., \& Shrinivasa, N. C. (2018). Segmentation of Arecanut Bunches using HSV Color Model. In 2018 International Conference on Electrical, Electronics, Communication, Computer, and Optimization Techniques (ICEECCOT), 1(1), 37-41.

Google Scholar $x^{\top}$

[31] Dhanesha, R., Naika, C. S., \& Kantharaj, Y. (2019). Segmentation of Arecanut Bunches using $\mathrm{YCgCr}$ Color Model. In 2019 1st International Conference on Advances in Information Technology (ICAIT), 1(1), 50-53.

Google Scholar $X^{\top}$

[32] Dhanesha, R., \& Naika, C. S. (2019). A Novel Approach for Segmentation of Arecanut Bunches Using Active Contouring. In Integrated Intelligent Computing, Communication and Security, 1(1), 677-682. Springer, Singapore.

Google Scholar $\chi^{7}$ 
[33] Siddesha, S., Niranjant, S. K., \& Aradhya, V. M. (2017). Segmentation of oil palm crop bunch from tree images. In 2017 International Conference on Smart Technologies for Smart Nation (SmartTechCon), 1(1), 1621-1626.

Google Scholar $X^{\nearrow}$

[34] Cecotti, H., Rivera, A., Farhadloo, M., \& Pedroza, M. A. (2020). Grape detection with convolutional neural networks. Expert Systems with Applications, 159(1), 1-9.

Google Scholar $\chi^{\top}$

[35] Liu, S., \& Whitty, M. (2015). Automatic grape bunch detection in vineyards with an SVM classifier. Journal of Applied Logic, 13(4), 643-653.

Google Scholar ${ }^{\top}$

[36] Aguiar, A. S., Magalhães, S. A., Dos Santos, F. N., Castro, L., Pinho, T., Valente, J., \& BoaventuraCunha, J. (2021). Grape bunch detection at different growth stages using deep learning quantized models. Agronomy, 11(9), 1-23.

Google Scholar ${ }^{\top}$

[37] Mohimont, L., Roesler, M., Rondeau, M., Gaveau, N., Alin, F., \& Steffenel, L. A. (2021). Comparison of Machine Learning and Deep Learning Methods for Grape Cluster Segmentation. In International Conference on Smart and Sustainable Agriculture, 1470(1), 84-102. Springer, Cham.

Google Scholar $\not 7$

[38] Rahman, A., Hellicar, A. (2014). Identification of mature grape bunches using image processing and computational intelligence methods, 2014 IEEE Symposium on Computational Intelligence for Multimedia, Signal and Vision Processing (CIMSIVP), 1(1), 1-6.

Google Scholar X

[39] Alharbi, A. G., \& Arif, M. (2020). Detection and Classification of Apple Diseases using Convolutional Neural Networks. In 2020 2nd International Conference on Computer and Information Sciences (ICCIS), 1(1),1-6

Google Scholar 7

[40] Prasad, S., Kumar, P., Hazra, R., \& Kumar, A. (2012). Plant leaf disease detection using gabor wavelets transform. In International Conference on Swarm, Evolutionary, and Memetic Computing, 1(1), 372-379. Springer, Berlin, Heidelberg.

Google Scholar $x^{\top}$

[41] Arivazhagan, S., Shebiah, R. N., Ananthi, S., \& Varthini, S. V. (2013). Detection of unhealthy region of plant leaves and classification of plant leaf diseases using texture features. Agricultural Engineering International: CIGR Journal, 15(1), 211-217.

Google Scholar ${ }^{\top}$

[42] Gavhale, K. R., Gawande, U., \& Hajari, K. O. (2014). Unhealthy region of citrus leaf detection using image processing techniques. In International Conference for Convergence for Technology, $1(1), 1-6$.

Google Scholar 7

[43] Singh, V., \& Misra, A. K. (2017). Detection of plant leaf diseases using image segmentation and soft computing techniques. Information processing in Agriculture, 4(1), 41-49.

Google Scholar $\not^{\top}$

[44] Jayamoorthy, S., \& Palanivel, N. (2017). Identification of Leaf Disease Using Fuzzy C-MEAN and Kernal Fuzzy C- MEAN and Suggesting the Pesticides. International Journal of Advanced Research in Science, Engineering and Technology, 4(5), 1-9.

Google Scholar $\chi^{\top}$

[45] Mokhtar, U., Ali, M. A., Hassenian, A. E., \& Hefny, H. (2015). Tomato leaves diseases detection An approach based on support vector machines. In 2015 11th International Computer Engineering Conference (ICENCO), 1(1), 246-250. IEEE. Google Scholar $\chi^{\top}$ 
[46] Omrani, E., Khoshnevisan, B., Shamshirb, S., Saboohi, H., Anuar, N. B., \& Nasir, M. H. N. (2014). Potential of radial basis function-based support vector regression for apple disease detection. Measurement, 55(1), 512-519

Google Scholar X

[47] Mamta, Y., \& Toran, V. (2016). Hybrid approach of neural network and genetic algorithm to recognize Black Mold disease in tomato. International Journal for Research in Applied Science and Engineering Technology, 4(5), 269-274.

Google Scholar $\chi^{\top}$

[48] Bhong, B. V. Pawar., \& S. Vijay. (2016). Study and Analysis of Cotton Leaf Disease Detection Using Image Processing. International Journal of Advanced Research in Science, Engineering and Technology, 3(2), 1447-1454.

Google Scholar ${ }^{\top}$

[49] Suresha, M., Shreekanth, K. N., \& Thirumalesh, B. V. (2017). Recognition of diseases in paddy leaves using knn classifier, 2nd International Conference for Convergence in Technology (I2CT),

Mumbai, 1(1), 663-666.

Google Scholar $\chi^{\top}$

[50] Hossain, E., Hossain, M. F., \& Rahaman, M. A. (2019). A Color and Texture Based Approach for the Detection and Classification of Plant Leaf Disease Using KNN Classifier, International Conference on Electrical, Computer and Communication Engineering (ECCE), Cox'sBazar, Bangladesh, 1(1), 1-6.

Google Scholar $x^{7}$

[51] Abdulridha, J., Ehsani, R., Abd Elrahman, A., \& Ampatzidis, Y. (2019). A remote sensing technique for detecting laurel wilt disease in avocado in presence of other biotic and abiotic stresses, Computers and electronics in agriculture, 156(1), 549-557.

Google Scholar $x^{7}$

[52] Ananthi N., Akshaya S., Aarthi B., Aishvarya J., Kumaran K. (2019). An Image Processing Based Fungus Detection System for Mangoes. International Journal of Innovative Technology and Exploring Engineering (IJITEE), 9(1), 3493-3499.

Google Scholar $\not$

[53] Nikhitha, M., Roopa Sri, S., \& Uma Maheswari, B. (2019). Fruit Recognition and Grade of Disease Detection using Inception V3 Model, 3rd International Conference on Electronics, Communication and Aerospace Technology (ICECA), 2019, 1(1), 1040-1043,

Google Scholar ${ }^{\top}$

[54] Wang, H., Mou, Q., Yue, Y., \& Zhao, H. (2020). Research on Detection Technology of Various Fruit Disease Spots Based on Mask R-CNN. IEEE International Conference on Mechatronics and Automation (ICMA), 1(1), 1083-1087.

Google Scholar $\chi^{\top}$

[55] Dhakate, M., \& Ingole, A. B. (2015). Diagnosis of pomegranate plant diseases using neural network, Fifth National Conference on Computer Vision, Pattern Recognition, Image Processing and Graphics (NCVPRIPG), 1(1), 1-4.

Google Scholar $X^{\top}$

[56] Nesarajan, D., Kunalan, L., Logeswaran, M., Kasthuriarachchi, S., \& Lungalage, D. (2020). Coconut Disease Prediction System Using Image Processing and Deep Learning Techniques, IEEE $4^{\text {th }}$ International Conference on Image Processing, Applications and Systems (IPAS), 1(1), 212217.

Google Scholar 7

[57] Abirami, S., \& Thilagavathi, M. (2019). Classification of Fruit Diseases using Feed Forward Back Propagation Neural Network, International Conference on Communication and Signal Processing (ICCSP), 1(1), 0765-0768.

Google Scholar 
[58] Samajpati, B. J., \& Degadwala, S. D. (2016). Hybrid approach for apple fruit diseases detection and classification using random forest classifier, International Conference on Communication and Signal Processing (ICCSP), 1(1), 1015-1019.

Google Scholar $X$

[59] Assunção, E., Diniz, C., Gaspar, P. D., \& Proença, H. (2020). Decision-making support system for fruit diseases classification using Deep Learning. International Conference on Decision Aid Sciences and Application (DASA), 1(1), 652-656.

Google Scholar $\chi^{\top}$

[60] Behera, S. K., Jena, L., Rath, A. K., \& Sethy, P. K. (2018). Disease Classification and Grading of Orange Using Machine Learning and Fuzzy Logic. International Conference on Communication and Signal Processing (ICCSP), 1(1), 0678-0682.

Google Scholar ${ }^{\top}$

[61] Monir Rabby, M. K., Chowdhury, B., \& Kim, J. H. (2018). A Modified Canny Edge Detection Algorithm for Fruit Detection \& Classification, 2018 10th International Conference on Electrical and Computer Engineering (ICECE), 1(1), 237-240.

Google Scholar $\chi^{7}$

[62] Doh, B., Zhang, D., Shen, Y., Hussain, F., Doh, R. F., \& Ayepah, K. (2019). Automatic Citrus Fruit Disease Detection by Phenotyping Using Machine Learning, 25th International Conference on Automation and Computing (ICAC), 1(1), 1-5. Google Scholar ${ }^{\top}$

[63] Al-Hiary, H., Bani-Ahmad, S., Reyalat, M., Braik, M., \& Alrahamneh, Z. (2011). Fast and detection and classification of plant diseases. International Journal of Computer Applications, 17(1), 31-38. Google Scholar $\chi^{\top}$

[64] Bargoti, S., \& Underwood, J. (2017). Deep fruit detection in orchards. IEEE International Conference on Robotics and Automation (ICRA), 1(1), 3626-3633

Google Scholar $\chi^{\top}$

[65] Ramesh Kestur., Avadesh Meduri., Omkar Narasipura MangoNet. (2019). A deep semantic segmentation architecture for a method to detect and count mangoes in an open orchard, Engineering Applications of Artificial Intelligence, 77(1), 59-69.

Google Scholar $\chi^{\top}$

[66] Awate, A., Deshmankar, D., Amrutkar, G., Bagul, U., \& Sonavane, S. (2015). Fruit disease detection using color, texture analysis and ANN, International Conference on Green Computing and Internet of Things (ICGCIoT), 1(1), 970-975.

Google Scholar ${ }^{\top}$

[67] Prathusha, P., Murthy, K. S., \& Srinivas, K. (2019). Plant Disease Detection Using Machine Learning Algorithms. In International Conference on Computational and Bio Engineering, 1(1), 213-220. Springer, Cham.

Google Scholar $\chi^{7}$

[68] Waghmare, H., Kokare, R., \& Dandawate, Y. (2016). Detection and classification of diseases of grape plant using opposite color local binary pattern feature and machine learning for automated decision support system. In $20163 \mathrm{rd}$ international conference on signal processing and integrated networks (SPIN), 1(1), 513-518.

Google Scholar $\chi^{\top}$

[69] Rozario, L. J., Rahman, T., \& Uddin, M. S. (2016). Segmentation of the Region of Defects in Fruits and Vegetables. International Journal of Computer Science and Information Security, 14(5), 399406.

Google Scholar $X^{7}$

[70] Behera, S. K., Rath, A. K., \& Sethy, P. K. (2021). Maturity status classification of papaya fruits based on machine learning and transfer learning approach. Information Processing in Agriculture, 8(2), 244-250. 
$\underline{\text { Google Scholar } \not}$

[71] Oppenheim, D., Shani, G., Erlich, O., \& Tsror, L. (2019). Using deep learning for image-based potatotuber disease detection. Phytopathology, 109(6), 1083-1087.

Google Scholar $x^{\top}$

[72] Dubey, S. R., \& Jalal, A. S. (2016). Apple disease classification using color, texture and shape features from images. Signal, Image and Video Processing, 10(5), 819-826.

Google Scholar $x^{\top}$

[73] Usha, S., Karthik, M., Jenifer, R., \& Scholar, P. G. (2017). Automated Sorting and Grading of Vegetables Using Image Processing. International Journal of Engineering Research and General Science, 5(6), 53-61.

Google Scholar ${ }^{\top}$

[74] Zeng, G. (2017). Fruit and vegetables classification system using image saliency and convolutional neural network. IEEE 3rd Information Technology and Mechatronics Engineering Conference (ITOEC), 1(1), 613-617.

Google Scholar ${ }^{\top}$

[75] Khadabadi, G. C., Kumar, A., \& Rajpurohit, V. S. (2015). Identification and classification of diseases in carrot vegetable using Discrete Wavelet Transform. International Conference on Emerging Research in Electronics, Computer Science and Technology (ICERECT), 1(1), 59-64. Google Scholar $x^{\top}$

[76] Phadikar, S., Sil, J., \& Das, A. K. (2013). Rice diseases classification using feature selection and rule generation techniques. Computers and electronics in agriculture, 90(1), 76-85.

Google Scholar ${ }^{\top}$

[77] Dubey, S. R., \& Jalal, A. S. (2012). Detection and Classification of Apple Fruit Diseases Using Complete Local Binary Patterns. Third International Conference on Computer and Communication Technology, 1(1), 346-351.

Google Scholar $\chi^{7}$

[78] Kumar, C. S., Jenifer, J., Vidhya, G., \& Vijayabhasker, R. (2021). Improving Vegetable Disease Detection using Modified K-Means Clustering Algorithm. International Journal of Scientific Research \& Engineering Trends, 7(2), 801-805.

Google Scholar $x^{\top}$

[79] Pawar, M. M., Bhusari, S., \& Gundewar, A. (2012). Identification of infected pomegranates using color texture feature analysis. International Journal of Computer Applications, 43(22), 30-34.

Google Scholar ${ }^{\top}$

[80] Ahmad Loti, N. N., Mohd Noor, M. R., \& Chang, S. W. (2021). Integrated analysis of machine learning and deep learning in chili pest and disease identification. Journal of the Science of Food and Agriculture, 101(9), 3582-3594.

Google Scholar $\chi^{\top}$

[81] Rauf, H. T., Saleem, B. A., Lali, M. I. U., Khan, M. A., Sharif, M., \& Bukhari, S. A. C. (2019). Acitrus fruits and leaves dataset for detection and classification of citrus diseases through machine learning. Data in brief, 26, 104340, 1(1), 1-7.

Google Scholar $\not^{7}$

[82] Yao, Q., Guan, Z., Zhou, Y., Tang, J., Hu, Y., \& Yang, B. (2009). Application of support vector machine for detecting rice diseases using shape and color texture features. In 2009 international conference on engineering computation, 1(1),79-83.

Google Scholar $x^{7}$

[83] Doh, B., Zhang, D., Shen, Y., Hussain, F., Doh, R. F., \& Ayepah, K. (2019). Automaticcitrus fruit disease detection by phenotyping using machine learning. In 2019 25th International Conference on Automation and Computing (ICAC), 1(1),1-5.

Google Scholar $\chi^{\top}$ 
[84] Patil, P. U., Lande, S. B., Nagalkar, V. J., Nikam, S. B., \& Wakchaure, G. C. (2021). Grading and sorting technique of dragon fruits using machine learning algorithms. Journal of Agriculture and Food Research, 4(1), 1-6.

Google Scholar ${ }^{\top} \quad$ CrossRefDOI $\chi^{\top}$

[85] Risdin, F., Mondal, P. K., \& Hassan, K. M. (2020). Convolutional Neural Networks (CNN) for Detecting Fruit Information Using Machine Learning Techniques. IOSR J. Comput. Eng., 22(2), 113.

Google Scholar X

[86] Malar, B. A., Andrushia, A. D., \& Neebha, T. M. (2021). Deep Learning based Disease Detection in Tomatoes. In 2021 3rd International Conference on Signal Processing and Communication (ICPSC), 1(1), 388-392. DOI: 10.1109/ICSPC51351.2021.9451731.

Google Scholar $X^{\top}$

[87] Mohanapriya, S., Efshiba, V., \& Natesan, P. (2021). Identification of Fruit Disease Using Instance Segmentation. In 2021 Third International Conference on Inventive Research in Computing Applications (ICIRCA), 1(1), 1779-1787.

Google Scholar $\nearrow^{\nearrow} \quad \underline{\text { CrossRefDOI } \chi^{\nearrow}}$

[88] Malathy, S., Karthiga, R. R., Swetha, K., \& Preethi, G. (2021). Disease Detection in Fruits using Image Processing. In 2021 6th International Conference on Inventive Computation Technologies (ICICT), 1(1), 747-752. Google Scholar ${ }^{\top}$

\section{CrossRefDOIX}

[89] Marani, R., Milella, A., Petitti, A., \& Reina, G. (2021). Deep neural networks for grape bunch segmentation in natural images from a consumer-grade camera. Precision Agriculture, 22(2), 387413.

Google Scholar ${ }^{\top}$

[90] Kurian, A., \& Peter, K. V. (2007). Commercial crops technology. New India Publishing, 8(1), 1386.

Google Scholar $\not 7$

[91] Chandra Mohanan, R., \& Babu, M. (2011). Integrated management of diseases in arecanut based cropping systems. Arecanut Based Cropping/Farming Systems. Central Plantation Crops Research Institute, Kasaragod. Published by Director Central Plantation Crops Research Institute (Indian Council of Agricultural Research) Kasaragod-671 124, Kerala, India, 1(1), 1-145.

Google Scholar X

[92] Chowdappa, P., Hegde, V., Chaithra, M., \& Thava Prakasa Pandian, R. (2016). Arecanut diseases and their Management. Indiall Journal of Arecanlll, Spices \& Medicinal Plants, 18 (4), 46-51. Google Scholar $\not$ '

[93] Gangadhara Naik, B., Maheswarappa, H. P., Nagamma, G., \& Latha, S. (2019). Management of fruit rot disease of arecanut (Areca catechu L.) caused by (Phytophthora meadii Mc Rae.). International Journal of Current Microbiology and Applied Sciences, 8(4), 837-84. Google Scholar $\chi^{7}$

[94] Balanagouda, P., Vinayaka, H., Maheswarappa, H. P., \& Narayanaswamy, H. (2021). Phytophthora diseases of arecanut in India: prior findings, present status and future prospects. Indian Phytopathology, 1(1), 1-12.

Google Scholar X'

[95] Sastry, M. N. L., \& Hegde, R. K. (1988). Control of fruit rot or koleroga disease of arecanut (Areca catechu L.). Tropical agriculture, 65(2), 150-152.

Google Scholar $\chi^{\top}$

[96] Narayanaswamy, H., Raju, J., \& Jayalakshmi, K. (2017). Management of fruit rot disease of arecanut incited by Phytophthora meadii. International journal of current microbiology andApplied Science, 6(7), 2824-2828.

Google Scholar X 
[97] Kasture, M. C., Dademal, A. A., More, S. S., \& Kadam, R. G. (2019). Effect of Boron Fortified Konkan Annapurna Briquettes on Yield and Nut Splitting of Arecanut in Coastal Konkan Region of Maharashtra. Journal of the Indian Society of Coastal Agricultural Research, 37(1), 14-18.

Google Scholar ${ }^{\top}$

[98] Ramesh, R., Maruthadurai, R., \& Singh, N. P. (2014). Management of fruit rot (Koleroga/Mahali) disease of Arecanut. ICAR Research Complex for Goa (Indian Council of Agricultural Research) Old Goa-403 402, Goa, India, 1(1), 1-2.

Google Scholar 7

[99] Hegde, G. M. (2015). Bio-efficacy of potassium phosphonate against nut rot disease of arecanut (Areca catechu L.) in the northern Karnataka of India. Sri Lanka Journal of Food and Agriculture, 1(2), 9-14.

Google Scholar $x^{\top}$

[100] Karpagam, D., Jansirani, R. (2019). SWOC Analysis and strategies for Promotion of Organic Paddy Farming in Cauvery Delta Zone of Tamil Nadu, India. International Journal of Current Microbiology and Applied Sciences (IJCMAS), 8(5), 427-433. Google Scholar $X^{\nearrow} \quad$ CrossRefDOI $X^{\nearrow}$

[101] Puneeth, B. R., \& Nethravathi, P. S. (2021). Paytm's Journey Towards Digital Payment in IndiaA Case Study. International Journal of Case Studies in Business, IT and Education (IJCSBE), 5(2), 125-141.

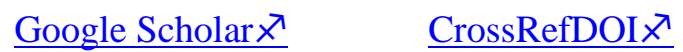

[102] Puneeth, B. R., \& Nethravathi, P. S. (2021). Bicycle Industry in India and its challenges-A Case Study. International Journal of Case Studies in Business, IT and Education (IJCSBE), 5(2), 62-74. Google Scholar \ CrossRefDOI $x^{\nearrow}$

[103] Murthy, A., \& Nethravathi, P. S. (2021). The Evolution of the E-Vehicle Industry and its Path Towards Setting up Dominance in Automobile Industry-A Case Study. International Journal of Case Studies in Business, IT and Education (IJCSBE), 5(2), 38-49.

Google Scholar $\chi^{\searrow} \quad \underline{\text { CrossRefDOI } \}$

$* * * * * * * * * *$ 